TITLE:

\title{
Rheological properties of ionic liquid solutions of xanthan
}

$\operatorname{AUTHOR}(S)$ :

Horinaka, Jun-ichi; Tanaka, Maki; Takigawa, Toshikazu

CITATION:

Horinaka, Jun-ichi ...[et al]. Rheological properties of ionic liquid solutions of xanthan. Colloid and Polymer Science 2015, 293(9): 27092712

ISSUE DATE:

2015-09

URL:

http://hdl.handle.net/2433/202569

\section{RIGHT:}

The final publication is available at Springer via http://dx.doi.org/10.1007/s00396-015-3718-1.; The full-text file will be made open to the public on 01 August 2016 in accordance with publisher's 'Terms and Conditions for Self-Archiving'.; この論文は出版社版でありません。引用の際には出版社版をご確認ご利用ください。; This is not the published version. Please cite only the published version. 


\section{Rheological properties of ionic liquid solutions of xanthan}

Jun-ichi Horinaka ${ }^{1}$ Maki Tanaka $^{1} \cdot$ Toshikazu Takigawa $^{1}$

${ }^{1}$ Department of Material Chemistry, Graduate School of Engineering, Kyoto University,

Nishikyo, Kyoto 615-8510, Japan

\section{Corresponding author: Jun-ichi Horinaka \\ E-mail: horinaka.junichi.5c@kyoto-u.ac.jp}

Tel: $+81-75-383-2454$

Fax: $+81-75-383-2458$ 
Abstract Dynamic viscoelasticity and steady shear flow of ionic liquid solutions of xanthan were examined. Concentrated solutions of xanthan were prepared using 1-butyl-3-methylimidazolium acetate as the solvent. The solutions exhibited the viscoelastic behavior typical of polymer solutions with the entanglement coupling. The absolute value of the complex viscosity and the steady shear viscosity agreed quite well, namely the Cox-Merz rule held, when the terminal flow behavior was observed in the dynamic viscoelasticity and the zero-shear viscosity was able to be determined. The molecular weight between entanglements for xanthan in the molten state was estimated to be $1.7 \times 10^{4}$, being significantly greater than that for cellulose 


\section{Introduction}

Xanthan has attracted a great deal of attention primarily because of the characteristic chain conformations in aqueous solutions [1-5]. First of all, xanthan just produced by the bacterium Xanthomonas campestris, which is referred to as the native xanthan, takes a rodlike helical conformation in water [3-6]. Heating the native xanthan brings about an order (helix)-to-disorder (coil) transition and the resultant flexible conformation is called the denatured form of xanthan. Moreover, the addition of salt to an aqueous solution of the denatured xanthan causes a disorder-to-order transition in the conformation. It has been reported that similar transition also occurs with a slow cooling of an aqueous solution of xanthan from a high temperature [1]. The sample after the disorder-to-order transition is termed the renatured xanthan, where the helical conformation is partly loosened in comparison to that of the native xanthan $[1,2,5]$. The change in the chain conformation described above of course affects the rheological properties of xanthan aqueous solutions. Therefore, in addition to the chain properties, the rheological properties have been extensively examined by many research groups $[5,7-9]$.

On the other hand, there are few studies on the chain properties and the rheological properties of xanthan dissolved in solvents other than water. In this regard, it is noteworthy that xanthan dissolved in cadoxen, a well-known solvent for cellulose, exists as flexible single chains at the 
temperature where xanthan in water with salt $\mathrm{NaCl}$ takes a rodlike conformation, although cadoxen, containing cadmium and ethylenediamine, is still an aqueous solvent [4]. This result indicates that xanthan in the disordered form could be obtained at various temperatures using specific solvents. Recently ionic liquids have become familiar as solvents for several polysaccharides including cellulose [10-13]. If xanthan is dissolved in an ionic liquid, xanthan might have the disordered conformation as the case of cadoxen mentioned above. However, to our knowledge, there is no information on ionic liquid solutions of xanthan, although the preparation and characterization of xanthan gels with an ionic liquid have been reported in the previous literature $[14,15]$.

With regard to the rheological properties, an interesting difference between aqueous and non-aqueous solutions of xanthan can be expected at high concentrations. Xanthan chains in an aqueous solution, namely in the ordered conformation, are known to cause self-association at high concentrations, so that liquid crystalline domains are formed above a certain concentration [7]. On the other hand, it is generally accepted that flexible polymers of high molecular weight in concentrated solutions tend to show the so-called entanglement coupling between polymer chains due to the topological constraints instead of self-association [16-20]. Previously we have prepared concentrated solutions of several polysaccharides, excluding xanthan, using ionic liquids and have shown that the solutions exhibit rheological behavior typical of polymer liquids 
containing the entanglement coupling without any chain aggregation [11,12,21-23]. Likewise, the rheological properties of ionic liquid solutions of xanthan might be totally different from those of the aqueous solutions at high concentrations.

Among several ionic liquids, we have found that 1-butyl-3-methylimidazolium acetate (BmimAc) dissolve xanthan up to the concentration where the entanglement coupling occurs. Hence, the rheological properties of the BmimAc solutions of xanthan have been examined in the current study. Furthermore, the entanglement coupling observed at high concentrations has been characterized in terms of the molecular weight between entanglements $\left(M_{\mathrm{e}}\right)$.

\section{Experimental}

Materials Xanthan (MP Biomedicals, USA) was dried in a vacuum oven at $80{ }^{\circ} \mathrm{C}$ overnight just before use. The ionic liquid solvent 1-butyl-3-methylimidazolium acetate (BmimAc) (BASF, Germany) was used without further purification. BmimAc solutions of xanthan were prepared in the following way. The powdery xanthan was added to BmimAc under vigorous stirring at room temperature, and the mixture was further stirred on a hot plate at $80^{\circ} \mathrm{C}$ for $1 \mathrm{~h}$. Then the mixture was left on the hot plate at $80^{\circ} \mathrm{C}$ overnight to dissolve xanthan completely. The concentration of xanthan $(c)$ ranged from $5.3 \times 10^{1}$ to $2.1 \times 10^{2} \mathrm{kgm}^{-3}\left(5\right.$ to $\left.20 \mathrm{wt}^{\%} \%\right)$. In calculating $c, 1.0 \times 10^{3}$ $\mathrm{kgm}^{-3}$ was assumed for the density of xanthan in the molten state, and $1.055 \times 10^{3} \mathrm{kgm}^{-3}$ was 
used for the density of BmimAc as the reported value of the manufacturer [12].

Measurements Dynamic viscoelasticity and steady flow measurements were performed with the rheometer ARES (now TA Instruments, USA) under nitrogen atmosphere. In both measurements, a cone-plate geometry with the diameter of $25 \mathrm{~mm}$ and the cone angle of $0.1 \mathrm{rad}$ was used. The angular frequency $(\omega)$ dependence of the storage modulus $\left(G^{\prime}\right)$ and the loss modulus $\left(G^{\prime \prime}\right)$ was obtained using the dynamic viscoelasticity measurement at $\omega$ ranging from 0.01 to $100 \mathrm{~s}^{-1}$ with the shear strain amplitude $(\gamma)$ of 0.1 . The value of $\gamma$ was determined so that the measurement could be performed in the linear viscoelasticity region. The above measurement was carried out at several temperatures $(T)$ from 20 to $100{ }^{\circ} \mathrm{C}$. The steady flow behavior was examined for the solutions of $c=5.3 \times 10^{1}$ and $1.1 \times 10^{2} \mathrm{kgm}^{-3}$ over the shear rate ( $\dot{\gamma}$ ) from 0.1 to $100 \mathrm{~s}^{-1}$ at $80{ }^{\circ} \mathrm{C}$.

\section{Results and discussion}

Figure 1 shows $G^{\prime}$ and $G^{\prime \prime}$ plotted against $\omega$ for the solutions of xanthan at $c=5.3 \times 10^{1}$ and $2.1 \times 10^{2} \mathrm{kgm}^{-3}$; namely, the lowest and the highest concentrations employed in this study. The frequency-temperature superposition principle holds for the solutions and these curves are the so-called master curves with the reference temperature $\left(T_{\mathrm{r}}\right)$ of $80^{\circ} \mathrm{C}$. The horizontal shift factor 
is represented by $a_{\mathrm{T}}$. The loss tangent $\left(\tan \delta ; \tan \delta=G^{\prime \prime} / G^{\prime}\right)$ is also plotted in the figure, although the data are limited to the region near each minimum. The low $\omega a_{\mathrm{T}}$ region where $G^{\prime}<G^{\prime \prime}$ indicates flow of the solution, although the terminal relation $G^{\prime \prime} \propto \omega$ is not completely attained within the $\omega a_{\mathrm{T}}$ range examined for the solution of $c=2.1 \times 10^{2} \mathrm{kgm}^{-3}$. The flow behavior shown in the figure appears to be typical of polymer solutions without any intermolecular aggregation. The higher $\omega a_{T}$ region, where $G^{\prime}>G^{\prime \prime}$, corresponds to the so-called rubbery plateau, although the actual $G^{\prime}$ curves still slope up gradually probably due to the polydispersity of the xanthan sample. The rubbery plateau arises from the entanglement coupling between xanthan chains in the solutions [17-20,24]; the plateau region for the solution of $c=2.1 \times 10^{2}$ $\mathrm{kgm}^{-3}$ is obviously wider than that for $c=5.3 \times 10^{1} \mathrm{kgm}^{-3}$, as expected. Master curves of $G^{\prime}$ and $G^{\prime \prime}$ for the BmimAc solutions of other concentrations have exhibited similar viscoelastic behavior to those in Figure 1.

Figure 2 shows $\log a_{\mathrm{T}}\left(T_{\mathrm{r}}=80^{\circ} \mathrm{C}\right)$ plotted against $1 / T$ for the solutions of $c$ from $5.3 \times 10^{1}$ to $2.1 \times 10^{2} \mathrm{kgm}^{-3}$. It is illustrated that $\log a_{T}$ at a given $T$ is almost the same regardless of $c$ and that all data points can be fitted by a straight line very well, as drawn in the figure, indicating that the $T$ dependence of $a_{\mathrm{T}}$ is represented by an Arrhenius-type equation within the conditions employed in this study.

It is well known that the Cox-Merz rule holds empirically for many polymer solutions with 
entanglement coupling, where the absolute value of the complex viscosity $\left(\left|\eta^{*}\right|\right)$ as the function of $\omega$ coincides with the steady shear viscosity $(\eta)$ as the function of $\dot{\gamma}$ [25]. To test this empirical rule, $\left|\eta^{*}\right|$ for the solutions of $c=5.3 \times 10^{1}$ and $1.1 \times 10^{2} \mathrm{kgm}^{-3}$ has been estimated from the data points in Figure 1 according to the definition

$$
\left|\eta^{*}\right|=\left[\left(\frac{G^{\prime}}{\omega}\right)^{2}+\left(\frac{G^{\prime \prime}}{\omega}\right)^{2}\right]^{\frac{1}{2}}
$$

and are plotted in Figure 3 together with $\eta$ from the steady flow measurement. It is obvious that the Cox-Merz rule holds for these solutions. Concerning aqueous solutions of xanthan, it has been reported that the application of the Cox-Merz rule are almost impossible due to the ordered structure of xanthan; it could be possible only for very dilute solutions compared with ours $[5,8,9]$. Although the reason why the Cox-Merz rule holds for many polymer solutions is still under investigation, the chain conformation might affect the applicability of that empirical rule to the xanthan solutions.

Since all the BmimAc solutions examined in this study have shown the rubbery plateau, as described above, the entanglement coupling of xanthan is characterized by $M_{\mathrm{e}}\left(\right.$ in $\mathrm{gmol}^{-1}$ ) in the following manner. The value of $M_{\mathrm{e}}$ is the average mesh size of the transient entanglement network and therefore can be evaluated from the analogy with the rubber elasticity as

$$
M_{\mathrm{e}}=\frac{10^{3} c R T}{G_{\mathrm{N}}^{0}}
$$


where $G_{\mathrm{N}}^{0}$ is the plateau modulus, which corresponds to the height of the rubbery plateau, and $R$ is the gas constant [17-19]. For sloping rubbery plateaus, we have defined $G_{\mathrm{N}}^{0}$ as the $G^{\prime}$ value at $\omega a_{\mathrm{T}}$ where $\tan \delta$ attains the minimum in the plateau regions [11,21]. According to the master curves in Figure 1, $G_{N}^{0}$ for the solutions of $c=5.3 \times 10^{1}$ and $2.1 \times 10^{2} \mathrm{kgm}^{-3}$ are obtained to be $5.1 \times 10^{2}$ and $7.0 \times 10^{3} \mathrm{~Pa}$, respectively. Thus $G_{N}^{0}$ and then $M_{\mathrm{e}}$ for the solutions of xanthan have been determined from the dynamic viscoelasticity data. The obtained values of $M_{\mathrm{e}}$ for xanthan in the BmimAc solutions are double-logarithmically plotted against $c$ in Figure 4. The data points are fitted by a straight line with the slope of -1 , because it is known that the relation of $M_{\mathrm{e}} \propto c^{-1}$ holds for the entanglement coupling of polymers in concentrated solutions $[17,19,24]$. It is demonstrated that the relation $M_{\mathrm{e}} \propto c^{-1}$ holds for the solutions of xanthan examined in this study. It might be interesting to estimate $M_{\mathrm{e}}$ for xanthan in the molten state $\left(M_{\mathrm{e}, \text { melt }}\right)$, namely $M_{\mathrm{e}}$ at $c=1.0 \times 10^{3} \mathrm{kgm}^{-3}$ (the assumed density of xanthan), using the relation $M_{\mathrm{e}} \propto c^{-1}$ given in Figure 4, although the molten state is not actually available for xanthan. Since $M_{\mathrm{e}, \text { melt }}$ especially is the material constant for polymers, there are many reported values of $M_{\mathrm{e}, \text { melt }}$ for polymers including our previous results for polysaccharides $[18,19,24]$. The straight line in Figure 4 gives $M_{\mathrm{e} \text {,melt }}$ of $1.7 \times 10^{4}$ for xanthan.

In the previous study, $M_{\mathrm{e}, \mathrm{melt}}$ for cellulose (cotton linters) has been estimated from the $M_{\mathrm{e}}$ values in the BmimAc solutions to be $2.9 \times 10^{3}$, which is much smaller than that for xanthan 
obtained in this study [26]. Since xanthan and cellulose have the same main chain of repeating glucose-ring units, the significant increase in $M_{\mathrm{e} \text {,melt }}$ for xanthan might be attributed to the long side chain, which is composed of mannose, glucuronic acid, and mannose-6-acetyl in series [6,27]; although how the side chain affects $M_{\mathrm{e} \text {,melt }}$ for xanthan cannot be mentioned in detail at the present stage. More comparison of the entanglement network between xanthan and cellulose can be made in terms of the number of repeating glucose-ring units between entanglements $\left(N_{\text {unit }}\right)$. The value of $N_{\text {unit }}$ is useful to compare the entanglement network among polysaccharides of different molecular structures. For the homopolysaccharide cellulose, $N_{\text {unit }}$ is simply calculated as the ratio $M_{\mathrm{e}, \text { melt }} / M_{\text {unit }}$ to be 18 , where $M_{\text {unit }}$ is the molecular weight of a repeating unit and is 162. Xanthan contains the side chain every two glucose-rings of the main chain, and about one-third of the terminal mannose residues is pyruvate substituted [2,6,27]. Hence, a repeating unit of xanthan corresponds to two glucose-ring units. Assuming the degree of pyruvation is one-third, $M_{\text {unit }}$ for xanthan becomes 888 and thus $N_{\text {unit }}$ is obtained as $2 M_{\text {e,melt }} / M_{\text {unit }}$ to be 38. The length between entanglement coupling points for xanthan is estimated to be significantly longer than that for cellulose. In the above discussion, the difference owing to the side chain between xanthan and cellulose is represented by $M_{\text {unit }}$, strictly $M_{\text {unit }}$ for xanthan versus $2 M_{\text {unit }}$ for cellulose, but it should be noted that $M_{\text {unit }}$ is not the exclusive factor that determines $M_{\text {e,melt }}$ or $N_{\text {unit }}[28]$. 


\section{Conclusion}

The rheological properties of BmimAc solutions of xanthan were examined with dynamic viscoelasticity and steady flow measurements. The solutions exhibited the viscoelastic behavior typical of simple polymer solutions with the entanglement coupling. The Cox-Merz rule held for the solution which showed the terminal flow behavior in the dynamic viscoelasticity and $\eta_{0}$ was determined. The entanglement coupling between xanthan appeared in the rheological data and $M_{\text {e,melt }}$ for xanthan was estimated to be $1.7 \times 10^{4}$.

\section{References}

1. Besio GJ, Leaveesley IM, Prud'homme RK (1987) J Appl Polym Sci 33:825

2. Holzwarth G, Prestridge EB (1977) Science 197:757

3. Sato T, Norisuye T, Fujita H (1984) Polym J 16:341

4. Takada Y, Sato T, Teramoto A (1991) Macromolecules 24:6215

5. Santore MM, Prud'homme RK (1990) Carbohyr Polym 12:329

6. Moorhouse R, Walkinshaw MD, Arnott S (1977) ACS Symposium Series 45:90

7. Lim T, Uhl JT, Prud'homme RK (1984) J Rheol 28:367

8. Rochefort WE, Middleman S (1987) J Rheol 31:337

9. Whitcomb PJ, Macosko CW (1978) J Rheol 22:493 
10. El Seoud OA, Koschella A, Fidale LC, Dorn S, Heinze T (2007) Biomacromolecules 8:2629

11. Horinaka J, Honda S, Takigawa T (2009) Carbohydr Polym 78:576

12. Horinaka J, Urabayashi Y, Takigawa T, Ohmae M (2013) J Appl Polym Sci 130:2439

13. Swatloski RP, Spear SK, Holbrey JD, Rogers RD (2002) J Am Chem Soc 124:4974

14. Izawa H, Kaneko Y, Kadokawa J (2009) J Mater Sci 19:6969

15. Izawa H, Kadokawa J (2010) J Mater Sci 20:5235

16. de Gennes PG (1979) Scaling concepts in polymer physics, Cornell University Press, New York

17. Ferry JD (1980) Viscoelastic properties of polymers, John Wiley \& Sons, New York

18. Doi M, Edwards SF (1986) The theory of polymer dynamics, Clarendon, Oxford

19. Masuda T, Toda N, Aoto Y, Onogi S (1972) Polym J 3:315

20. Onogi S, Masuda T, Kitagawa K (1970) Macromolecules 3:109

21. Horinaka J, Yasuda R, Takigawa T (2011) J Polym Sci B Polym Phys 49:961

22. Horinaka J, Yasuda R, Takigawa T (2012) J Appl Polym Sci 123:3023

23. Horinaka J, Okuda A, Yasuda R, Takigawa T (2012) Colloid Polym Sci 290:1793

24. Nemoto N, Ogawa T, Odani H, Kurata M (1972) Macromolecules 5:641

25. Cox WP, Merz EH (1958) J Polym Sci 28:619

26. Horinaka J, Urabayashi Y, Takigawa T, Cellulose DOI 10.1007/s10570-015-0670-7 
1

2

3

4

5

6

7

8

9

10

11

12

13

14

15

16

17

18

19

20

21

22

23

24

25

26

27

28

29

30

31

32

33

34

35

36

37

38

39

40

41

42

43

44

45

46

47

48

49

50

51

52

53

54

55

56

57

58

59

60
27. Jansson PE, Kenne L, Lindberg B (1975) Carbohydr Res 45:275

28. Fetters LJ, Lohse DJ, Richter D, Witten TA, Zirkel A (1994) Macromolecules 27:4639 
Figure Captions

Fig. 1 Master curves of $\omega$ dependence of $G^{\prime}$ and $G^{\prime \prime}$ for $5.3 \times 10^{1}$ and $2.1 \times 10^{2} \mathrm{kgm}^{-3}$ solutions of xanthan at $T=80^{\circ} \mathrm{C}$. The $\tan \delta$ curve is also included.

Fig. 2 Shift factor for solutions of xanthan of $c$ from $5.3 \times 10^{1}$ to $2.1 \times 10^{2} \mathrm{kgm}^{-3}$ plotted against the reciprocal of $T$. All data points fall on a single line

Fig. 3 Comparison of $\left|\eta^{*}\right|$ and $\eta$ for the solutions of xanthan at $c=5.3 \times 10^{1}$ and $1.1 \times 10^{2}$ $\mathrm{kgm}^{-3}$ at $T=80^{\circ} \mathrm{C}$.

Fig. 4 Double-logarithmic plot of $M_{\mathrm{e}}$ against $c$ for xanthan in the BmimAc solutions. The straight line is the best fit with the slope of $-1 . M_{\mathrm{e}, \text { melt }}$ for xanthan are determined as $M_{\mathrm{e}}$ at $c=1 \times 10^{3} \mathrm{kgm}^{-3}$. 
Fig. 1

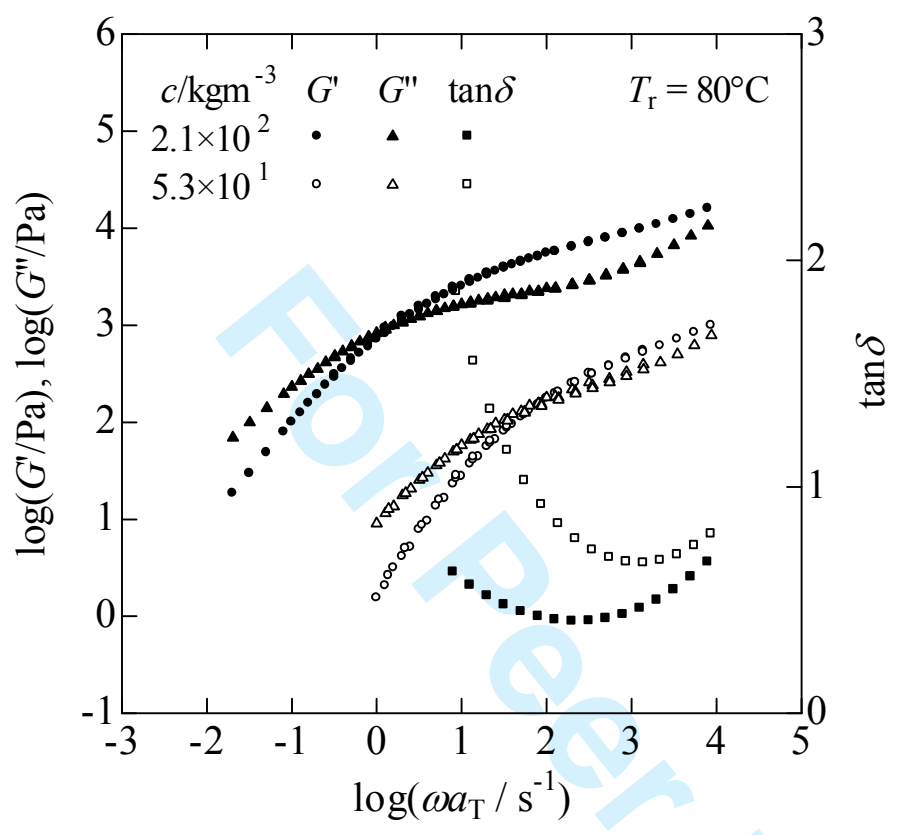


Fig. 2

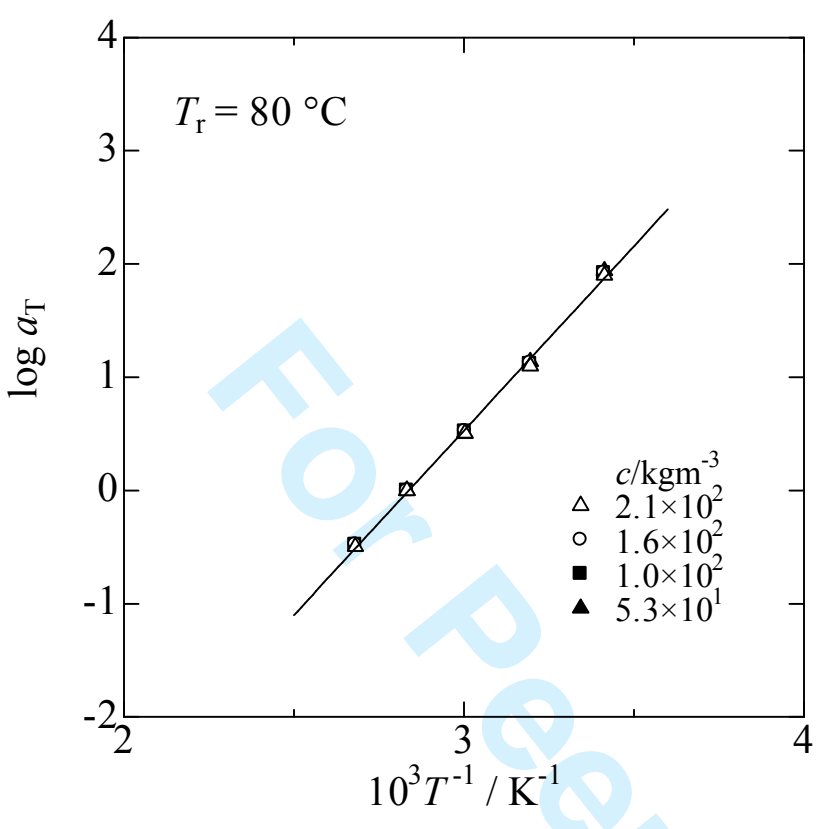

30

31

32

33

34

35

36

37

38

39

40

41

42

43

44

45

46

47

48

49

50

51

52

53

54

55

56

57

58

59 
Fig. 3

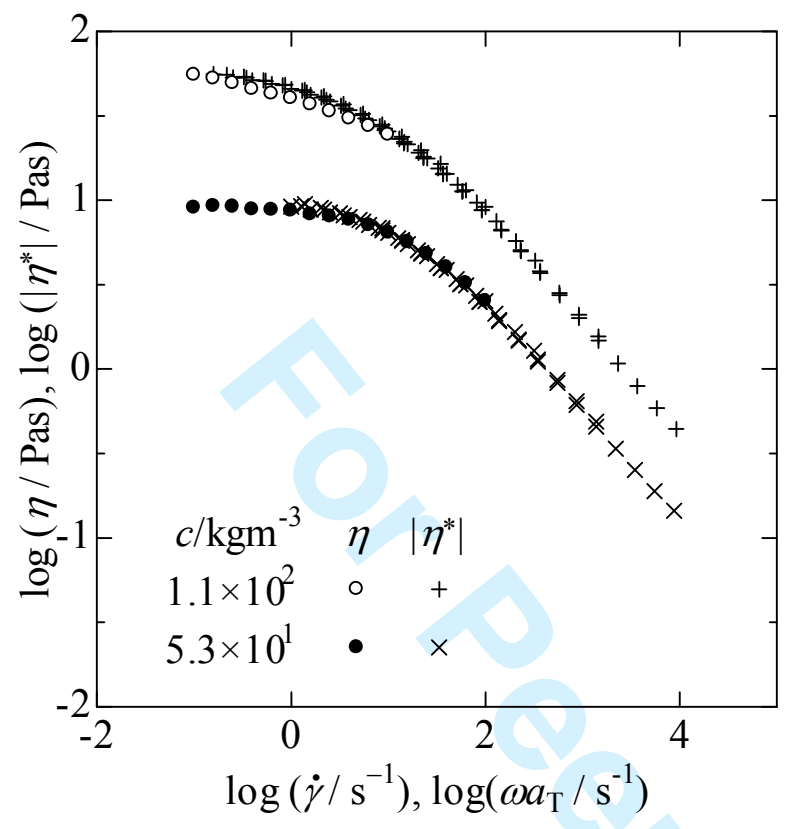

30

31

32

33

34

35

36

37

38

39

40

41

42

43

44

45

46

47

48

49

50

51

52

53

54

55

56

57

58

59 
Fig. 4

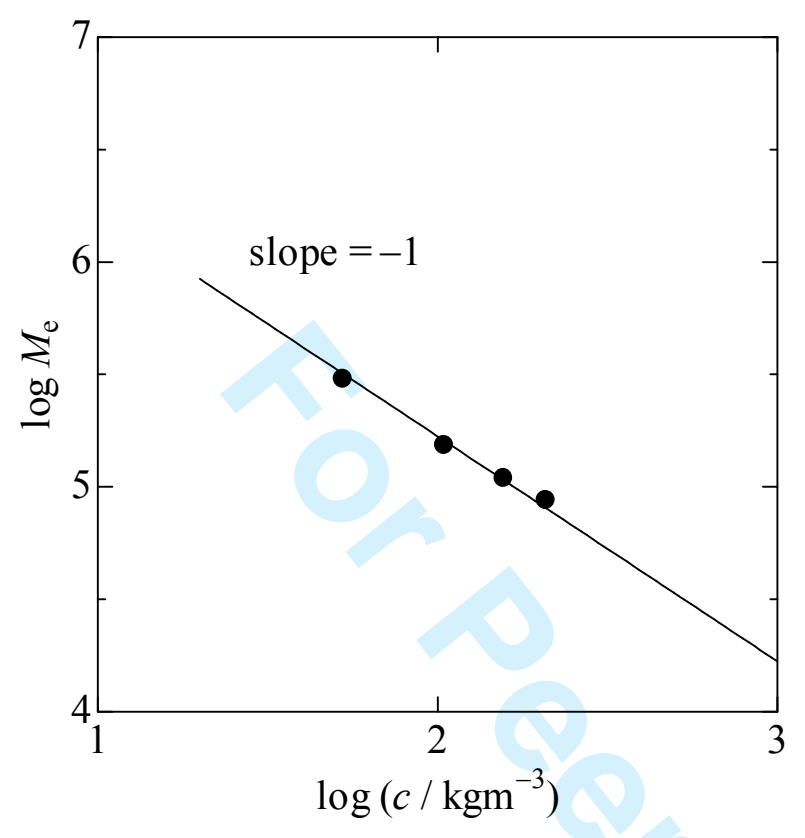

30

31

32

33

34

35

36

37

38

39

40

41

42

43

44

45

46

47

48

49

50

51

52

53

54

55

56

57

58

59

60 